Journal of Current and Advance Medical Research

July 2017, Vol. 4, No. 2, pp. 63-67

http://www.banglajol.info/index.php/JCAMR

ISSN (Print) 2313-447X

ISSN (Online) 2413-323X

DOI: http://dx.doi.org/10.3329/jcamr.v4i2.36359

Case Report

OPEN ACCESS

\title{
A Case Report On Cutaneous Leiomyosarcoma with Review of Literatures
}

\author{
Mahbuba Begum ${ }^{1}$, Mohammad Sharif Hossain ${ }^{2}$ \\ ${ }^{1}$ Assistant Professor, Department of Surgery, Medical College for Women and Hospital, Uttara model town, Dhaka, Bangladesh; \\ ${ }^{2}$ Department of Surgery, Medical College for Women and Hospital, Uttara model town, Dhaka, Bangladesh
}

[Received: 20 January 2017; Accepted: 31 March 2017; Published on: 1 July 2017]

\section{Abstract}

Cutaneous leiomyosarcoma represents superficial form of leiomyosarcoma which is a rare mesenchymal tumour originating from smooth muscle cells. Clinically cutaneous leiomyosarcoma can be confused with other cutaneous tumour and needs histopathology and immunohistochemistry to confirm the diagnosis. As it is an uncommon malignant neoplasm we encouraged reporting this case who presented to our hospital as an irregular nodular cutaneous lump of short duration in her right thigh. After local excision histopathological examination of excised specimen established the diagnosis of cutaneous leiomyosarcoma. A brief review of literature is also included here. [Journal of Current and Advance Medical Research 2017;4(2):63-67]

Keywords: Cutaneous Leiomyosarcoma; mesenchymal tumour; Primary cutaneous leiomyosarcoma

Correspondence: Dr. Mahbuba Begum, Assistant professor, Department of surgery, Medical College for Women and Hospital, Uttara model town, Dhaka-1230, Bangladesh; Email: mahbuba44k@gmail.com; Cell no.: +8801712721835

Cited As: Begum M, Hossain MS. A Case Report On Cutaneous Leiomyosarcoma with Review of Literatures. Journal of Current and Advance Medical Research 2017;4(2):63-67

Conflict of Interest: None

Contributions to authors: Begum M \& Hossain MS have involved in the, history taking, patient management as well as the manuscript writing.

Copyright: (02017. Begum \& Hossain. Published by Journal of Current and Advance Medical Research. This article is published under the Creative Commons CC BY-NC License (https://creativecommons.org/licenses/by-nc/4.0/). This license permits use, distribution and reproduction in any medium, provided the original work is properly cited, and is not used for commercial purposes.

\section{Introduction}

Primary cutaneous leiomyosarcoma is a rare soft tissue tumour and also an unusual form of skin malignancy. It arises from erector pili muscle of the hair follicles and sweat glands in dermis and accounts for $1 \%$ to $3 \%$ of all soft tissue sarcomas ${ }^{1}$. Clinical presentation varies from person to person and is not always suggestive of malignancy. Histopathological examination is the only definitive mode to reach diagnosis. Immunohistochemistry also poses a great role to diagnose cutaneous leiomyosarcoma. Recommended treatment is surgical and wide local excision constitutes the current treatment of choice while radiotherapy and chemotherapy may be considered in high grade lesion $^{2}$. Here we report a young lady presented with cutaneous leiomyosarcoma in her right thigh. Preoperatively her diagnosis was inconclusive but after surgical excision histopathology confirmed the diagnosis. She received adjuvant chemotherapy. No recurrence is noted till now following operation.

\section{Case Presentation}

A 40 year old lady, a housewife presented to us with a five months history of a painless lump in her right thigh which was increasing in size. She had no problem with limb movement and no other medical comorbidities. Local examination revealed an irregular exophytic mass in middle part of 
anteromedial aspect of her right thigh. It was about $5 \times 4.5 \mathrm{~cm}$ in size, non-tender, irregular surface, variable in consistency, well defined irregular margin; overlying skin was involved mostly with erythema having subcutaneous extension partly but not fixed to underlying muscle.

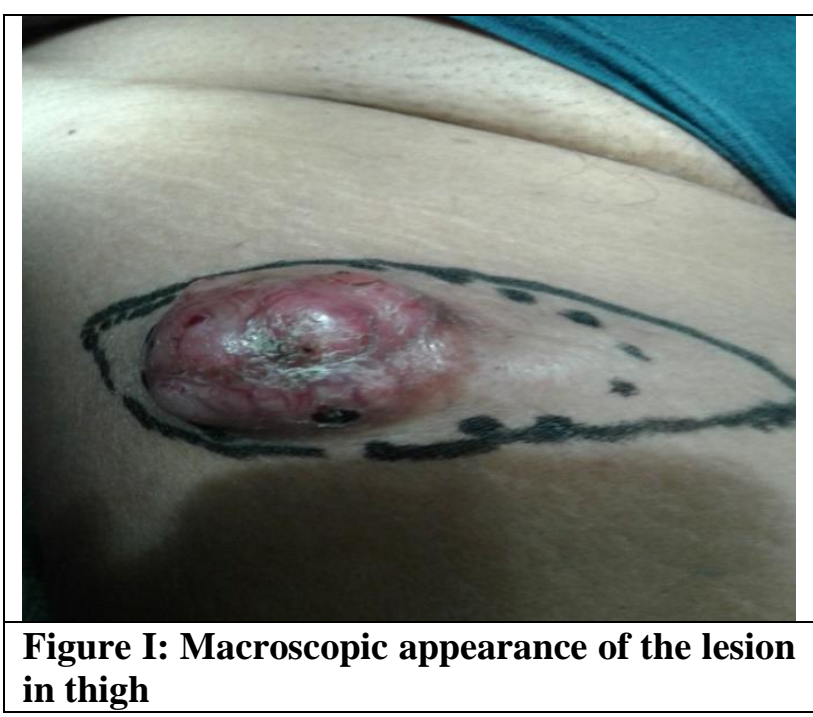

Her general and systemic examination appeared unremarkable. Soft tissue ultrasound scan of her right thigh revealed dermal mass with hypoechoic and heterogenous echo structure. FNAC of the lump was inconclusive. Her routine investigations were within normal limit. Clinically the lump was suspicious to us; we planned for local excision of lump. It was not adherent to underlying fascia or surrounding tissue, so it was easily excised out with $1 \mathrm{~cm}$ surrounding healthy margin. After undermining the skin and subcutaneous tissue wound was closed primarily.

Her postoperative period was uneventful. Wound appeared healthy following removal of stitches. Histopathology of excised lump confirmed leiomyosarcoma composed of malignant spindle to anaplastic cells with focal interlacing bundles appearance and areas of necrosis, haemorrhage with involvement of whole dermis. Resection margins were free from tumour infiltration. The patient was referred to consultant oncologist; she received 3 cycles of chemotherapy. Till date she is of good health.

\section{Discussion}

Leiomyosarcoma of soft tissue account for approximately 5.0 to $10.0 \%$ of all soft tissue sarcomas $^{3}$. Leiomyosarcoma of the skin includes superficial leiomyosarcoma arising from cutaneous structures and metastatic leiomyosarcoma that derives from distant visceral sites ${ }^{4}$.

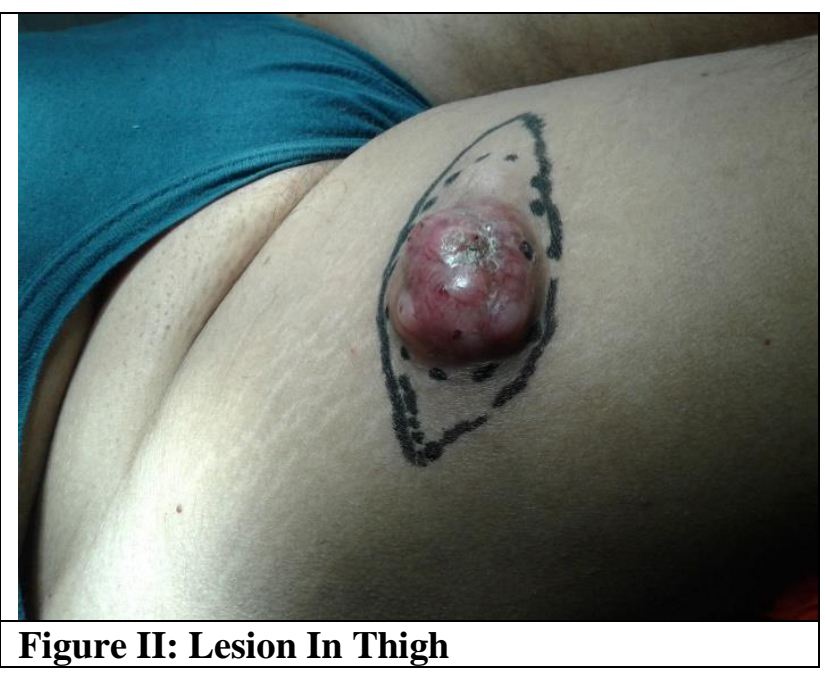

Superficial leiomyosarcoma is classically classified as cutaneous leiomyosarcoma and subcutaneous leiomyosarcoma depending on histological features and biological behavior. Cutaneous leiomyosarcoma develops in the dermis usually from smooth muscle cells surrounding the sudoriferous glands and the erector muscle of hair and can extend to the superficial soft tissue. Subcutaneous leiomyosarcoma is more deeply situated and arises from smooth muscle of vessels ${ }^{5}$. This tumour is prevalent between $5^{\text {th }}$ to $7^{\text {th }}$ decades of life; it is very rare in childhood ${ }^{5}$. A male predominance was noted with male to female ratio of 2:1. Higher incidence in Caucasian race is reported in literature ${ }^{5}$. The causes of cutaneous leiomyosarcoma are unknown. The most common predisposing factors reported are trauma and radiotherapy ${ }^{6}$. Other factors suggested are solar exposure, burns, chemical agents, vaccines, tick bites, venous stasis or lupus, tattooing ${ }^{7}$ and close to the site of titanium pacemaker ${ }^{8}$. No such etiological correlation was noted in our patient. Occurrence of this tumour in such situation could be a coincidence. However, it was suggested that chronic inflammation or irritation could be a possible etiopathogenic mechanism ${ }^{8}$. Malignant transformation of a leiomyoma has also been reported ${ }^{9}$.

Cutaneous leiomyosarcoma is usually located on the extremities with a predilection for hair bearing surfaces ${ }^{6}$. Other anatomical sites for its location are trunk, genitalia, head, neck, face, and buttock. Atypical sites are penis, breast, orbit and the external auditory canal $^{6}$. Clinically the lesions appear as solitary nodular cutaneous lump ranging from 0.4 to $6 \mathrm{~cm}^{10}$. Larger size up to19 $\mathrm{cm}$ was reported in the literature ${ }^{2}$. Larger tumour $(>5 \mathrm{~cm})$ are considered to have worst prognosis in terms of recurrence and metastasis ${ }^{2}$. Usually cutaneous 
leiomyosarcoma are sessile, sometimes pedunculated, lobulated or umbilicated ${ }^{5-6}$.

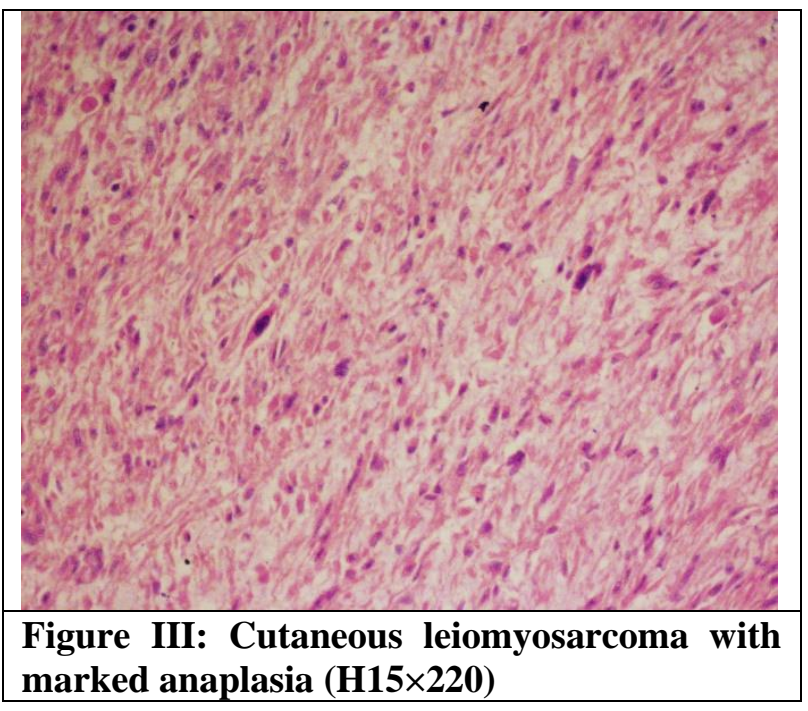

Lesions are firm, colored from pink red to deep red, violet, tan, brown, black or hypopigmented ${ }^{6,11}$. They are of irregular contour; surface can be smooth, indurated, ulcerated, scaly, verrucous or hemorrhagic ${ }^{10}$. Cutaneous leiomyosarcoma is localized mainly in the dermis but can extend into the superficial subcutaneous tissue or epidermis while subcutaneous leiomyosarcoma usually arises between junction of dermis and hypodermis and mostly exists in subcutaneous tissue ${ }^{11-12}$. Histologically cutaneous leiomyosarcoma characterized by dermal proliferation of highly cellular interlacing fascicles of spindle cells with blunt ended nuclei giving cigar appearance and multiple mitotic figures ${ }^{5-6}$.

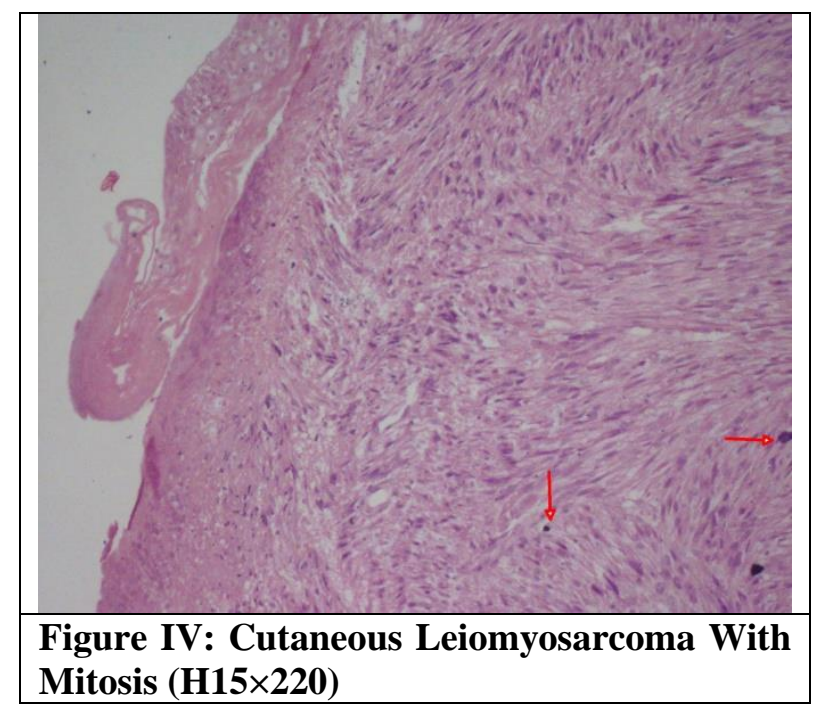

Tumor borders show an irregular aspect with smooth muscle cells through collagen and adipocytes these features explain why a radical surgical excision is often difficult ${ }^{13}$. Conversely subcutaneous leiomyosarcoma is a well circumscribed mass with a pseudocapsule of surrounding collagen ${ }^{11}$. Histopathological diagnosis of cutaneous leiomyosarcoma requires an appearance of dense cellularity with eosinophilic cytoplasm, nuclear atypia, pleomorphism with at least one mitotic figure per 5 to $10 \mathrm{HPF}^{11}$.

Careful scrutiny of cytological details in multiple sections of specimen along with clinic-pathological correlation is mandatory to avoid misinterpretation as benign smooth muscle proliferation like leiomyomas and smooth muscle hamartomas ${ }^{6}$. A number of benign and malignant tumour like lesions are difficult to distinguish from cutaneous leiomyosarcoma namely dermato-fibro-sarcoma protuberans, cysts, lipoma, neurofibroma, atypical fibroxanthoma, basal cell carcinoma, squamous cell carcinoma, pyogenic granuloma, spindle cell angiosarcoma, malignant histiocytofibroma and schwannoma ${ }^{6-12}$. Recommended treatment is surgical removal. Preoperative diagnostic workup plays limited role. Fine needle aspiration cytology of the lump is mostly inconclusive due to subtle microscopic features. Shave biopsy or punch biopsy may give better result ${ }^{1}$.

Soft tissue ultrasound scan will confirm a soft tissue mass and often show the plane the mass is in, however it is less helpful in determining precise anatomical relationship or nature of lesion ${ }^{14}$. X-ray of affected part may exclude bony involvement. MRI can confirm suprafascial character and extension of the lesion and reveal no clear relationship with the surrounding muscle and bone ${ }^{2}$. Chest X-ray, computed tomography scan of chest and abdomen, total bone scan, whole body positron emission tomography can exclude the presence of systemic lesions or metastasis ${ }^{1-2}$. As metastases have rarely been described in patient with cutaneous leiomyosarcoma the utility of preoperative staging appears to add minimal clinical impact ${ }^{13}$.

Immunohistochemistry demonstration of muscle specific antigens facilitates differentiation of these smooth muscle tumours from other forms of spindle cell tumour ${ }^{15}$. In this regard common markers are actin, vimentin and desmin. We did not go for extensive preoperative evaluation of the patient except for clinical fitness for operation. Local surgical excision is the proposed treatment for this lesion. Range of excisional margin and depth play important role in patient management as local excision without adequate margins lead to recurrence and increases the risk for metastatic and possibly fatal disease ${ }^{6}$. Surgical excisions are of 
intra-lesion, marginal and wide in types ${ }^{16}$. Surgical guidelines for both cutaneous and subcutaneous leiomyosarcoma despite their different prognostic entities are recommended as 3 to $5 \mathrm{~cm}$ margin excision including subcutaneous fat and deep fascia ${ }^{17}$. Jensen et $\mathrm{al}^{18}$ described their only patient with local recurrence because of inadequate margin excision. Massi et $\mathrm{a}^{19}$ in a retrospective study of 62 patients with extremity soft tissue leiomyosarcoma reported that seven out of the nine patients who had developed one or more local recurrences had been surgically treated with either marginal or intralesion excision. Likewise Svarvar et $\mathrm{al}^{16}$ in a large study of 225 patients with leiomyosarcoma showed that the inadequate local treatment was the main risk factor for local recurrence. Some authors ${ }^{5,12}$ suggest that limited margins are sufficient to the treatment of cutaneous form with low grade malignancy whereas wide excision is applicable for high grade malignancies and subcutaneous ones. Wide surgical excision may require some sort of reconstructive procedures and a challenge to functional and an esthetic point of view ${ }^{5}$. Mohs micrographic operation with narrow surgical margins is another method of treatment to ensure complete tumour removal. This procedure showed a considerable reduction in the risk of local recurrence, preservation of normal tissue and ensures amore rewarding functional and aesthetic result ${ }^{4}, 17$. Cryosurgery has also been used in elderly patient ${ }^{10}$. Adjuvant therapies include radiotherapy and chemotherapy. It is reported that adjuvant therapies are generally reserved for subcutaneous and deep tumours with a high grade malignancy with dimensions greater than $5 \mathrm{~cm}$ and with positive surgical margins ${ }^{5}$. Relapses usually occur within first to fifth year; lung is the common site for metastasis and involvement of regional lymph nodes is less common ${ }^{5}$. For cutaneous leiomyosarcoma local relapse ranges from 6.0 to $50.0 \%$ and rarely metastasize ${ }^{5-6}$. However, risk of local relapse is higher in subcutaneous leiomyosarcoma and range from 40.0 to $70.0 \%$ with metastasis in 30.0 to $60.0 \%$ of cases and risk of death is about 30.0 to $40.0 \% 5$. Prognostic factors for poor results are tumour size $>5 \mathrm{~cm}$, deep involvement with fascia, inadequate surgical excision, high grade malignancy and advanced tumour staging ${ }^{2,6}$. Sufficient therapy in situation of local relapse is simple re-excision suggested in literatures ${ }^{1}$. Deneve et al $^{1}$ in their retrospective study of 33 patients of which a total of $94 \%$ of patients underwent surgical resection with a median margin of $1 \mathrm{~cm}$; only $3 \%$ showed positive margins treated subsequently by re-excision and radiotherapy; no patient received adjuvant chemotherapy. In their study no local or regional recurrences or distant metastasis or mortality were present. In our case we excised the lump with $1 \mathrm{~cm}$ margin of clearance which showed no tumour infiltration on histopathological examination.

\section{Conclusion}

Cutaneous leiomyosarcoma is although a rare tumor may be confused with other cutaneous lesions both benign and malignant so precise histological scrutinization with clinicopathological correlation is mandatory to confirm diagnosis.

Immunohistochemistry may be utilized to reach a diagnosis or to rule out of the spindle cell tumour. As it is low grade malignancy having favourable therapeutic outcome adequate treatment canbe offered after proper timely diagnosis. Surgical treatment is the best modality of treatments for cutaneous leiomyosarcoma with special focus on careful and wide meticulous excision. However review of literatures revealed use of variable ranges of excision margins with mixed outcome. Benefit of use of adjuvant radiotherapy and chemotherapy is controversial and further study is recommended. Long term follow up for at least 5 years is advocated to note local relapses.

Acknowledgements: Prof. Mohammad Kamal, Professor and Chairman of Pathology, Bangabandhu Sheikh Mujib Medical University, Dhaka, Bangladesh for helping with pictures of slides.

\section{References}

1. Deneve JL, Messina JL, Bui MM, Marzban SS, Letson GD, Cheong D, Gonzalez RJ, Sondak VK, Zager JS. Cutaneous leiomyosarcoma: treatment and outcomes with a standardized margin of resection. Cancer Control. 2013;20(4):307-12

2. Angeloni M, Muratori F, Magarelli N, Chalidis BE, Ricci R, Rossi B, Maccauro G. Exophytic growth of a neglected giant subcutaneous Leiomyosarcoma of the lower extremity. A case report. In International Seminars in Surgical Oncology 2008;5(1):11

3. Weiss SW, Goldblum JR. Enzinger and Weiss's soft tissue tumours. $4^{\text {th }}$ ed. Philadelphia: Mosby - Harcourt; 2001. P.72748

4. Annest NM, Grekin SJ, stone MS, Messingham MJ. Cutaneous leiomyosarcoma: a tumour of the head and neck. Dermatol surg. 2007; 33(5): $628-633$

5. Fania L, Ghitti F, Manca R, Feliciani C, Guerriero G. Cutaneous leiomyosarcoma arising after $\mathrm{CO} 2$ laser therapy of an unknown lesion: case report and review of literature. Clinical dermatology 2014;2(4):166-172

6. Faten Limaiem MD, Ines Chelly MD, Selma Bellil MD, Amina Mekni MD, Kchir Nidhameddine MD, Slim Haouet MD, Khadija Bellil MD, Moncef Zitouna MD. Primary cutaneous leiomyosarcoma: a histological and immunohistochemical study of 4 cases. Egyptian Dermatology Online Journal. 2007;3:1

7. West CC, Morritt AN, Pedelty L, Lam DG. Cutaneous leiomyosarcoma arising in a tattoo-'a tumour with no humour'. Journal of Plastic, Reconstructive \& Aesthetic Surgery. 2009;62(5):e79-80 
8. González-Vela MC, Val-Bernal JF, Rubio S, Olalla JJ, González-López MA. Cutaneous leiomyosarcoma developing on a pacemaker pocket. Dermatologic Surgery. 2009;35(5):863-7

9. Tellechea O, Reis JP, Freitas JD, Masson P, Poiares A. Cutaneous leiomyosarcoma developing on a piloleiomyoma. $\mathbf{J}$ Dermatol. 1993;3:28-32

10. Kuflik JH, Schwartz RA, Rothenberg J. Dermal leiomyosarcoma. Journal of the American Academy of Dermatology. 2003;48(5):S51-3

11. Liao WC, Lin JT, Yeh FL, Ma H, Shen BH, Chen JH, Fang RH, Chen WY. Primary cutaneous leiomyosarcoma. Journal of plastic, reconstructive and aesthetic surgery 2003;12(1):1-9 12. Holst VA, Hopkins JM, Elenitsas R. Cutaneous smooth muscle neoplasm: clinical features, histologic findings and treatment option. J Am Acad Dermatol 2002;46(4):477-94

13. Kaddu S. Beham A, Cerroni L, Fuchsu H, Salmhofer W, et al. Cutaneous leiomyosarcoma. Am J surgpathol. 1997;21(9 ):979-87
14. WWW. Surgwiki.Com. Soft tissue tumours. Jan 2012

15. swanson PE, Stanley MW, Scheithauer BW, et al. Primary cutaneous leiomyosarcoma: a histological and immunehistochemical study of 9 cases with ultra-structural correlation. J Cutan Pathol 1988;15 : 129-41

16. Svarvar C, Bohling T, Berlino, Gustafson P et al. Clinical course of nonvisceral soft tissue leiomyosarcoma in 225 patients from the Scandinavian sarcoma group. Cancer 2007;109(2):282-91

17. Bernstein SC, Roenigk RK. Leiomyosarcoma of the Skin: treatment of 34 cases. Dermatol Surg 1996;22:631-5

18. Jensen ML, Jensen OM, Michalski W et al. Intradermal and subcutaneous leiomyosarcoma: a clinic-pathological and immune-histochemical study of 41 cases. J Cutan Pathol 1996; 23:458-63

19. Massi D, Franchi A, Alos L, Cook M, Di Palma S, Enguita AB, Ferrara G, Kazakov DV, Mentzel T, Michal M, Panelos J. Primary cutaneous leiomyosarcoma: clinicopathological analysis of 36 cases. Histopathology. 2010;56(2):251-62 